\title{
Clinical Effects of Edaravone in Stroke: A Review
}

\author{
Anisha Thomas*, Anuja Solomon, Tanushree MN, Akhila S Arjun \\ Department of Pharmacy Practice, Bapuji Pharmacy College, Davangere, Karnataka, INDIA.
}

\begin{abstract}
Edaravone, a free radical scavenger, is the only neuroprotective agent for ALS. Free radicals play a crucial role in brain ischemic injury by exacerbating membrane damage through peroxidation of unsaturated fatty acids of cell membrane, leading to neuronal death and brain edema. Free radicals have been implicated in stroke pathophysiology as pivotal contributors to cell injury. After brain damage, caused by either ischemic or hemorrhagic stroke, physiological systems involved in the removal of the excess of free radicals are impaired and the formation of free radicals is increased. This study aims to evaluate the safety and efficacy of edaravone, a novel free radical scavenger, in a group of Indian patients of acute ischemic stroke. However, its actions on pro-inflammatory responses under stroke are still understudied. This data suggests that free radical scavengers may reduce systemic inflammatory responses under acute stroke conditions and therefore, oxidative stress can be still a viable target for acute stroke therapy. Efficacy of edaravone range from large significant clinical improvements to only modest improvements in clinical function measured using standard stroke scales when administered 6-72 $\mathrm{h}$ following an ischemic stroke. With almost 17 years of edaravone clinical experience, a few adverse events including acute renal failure have been noted. Edaravone treatment was safe and effective in providing early and sustained neurological improvement in patients with acute ischemic stroke.
\end{abstract}

Key words: Edaravone, Stroke, Free radical, ALS, Neuroprotective, Ischemic stroke.

\section{INTRODUCTION}

Free radicals play a crucial role in brain ischemic injury by exacerbating membrane damage through peroxidation of unsaturated fatty acids of cell membrane, leading to neuronal death and brain edema. Free radicals have been implicated in stroke pathophysiology as pivotal contributors to cell injury. Edaravone (MCI-186, 3-methyl-1-phenyl-2-pyrazoline-5-one), a novel free radical scavenger, prevents vascular endothelial injury, delays neuronal death in transient cerebral ischemia and ischemic brain edema, inhibits activation of lipoxygenase pathway in the arachidonic acid cascade and peroxidation of the phosphatidylcholine liposomal membrane in vitro. ${ }^{1}$ Recanalization and neuroprotection have been mainly targeted for the specific treatment of acute ischemic stroke. A number of Japanese studies have shown it to be efficacious in patients with acute ischemic stroke. ${ }^{2}$ However, there has been no data available on the effect of edaravone in patients with acute ischemic stroke in the Indian subcontinent. In a randomized controlled trial, patients with acute ischemic stroke who were treated with edaravone had improved functional outcomes. Edaravone was approved for the treatment of acute cerebral infarction within $24 \mathrm{~h}$ of the onset of symptoms by the Japanese Ministry of Health, Labour and Welfare and became available in Japan in June 2001. Free radicals activate coagulation. Polymorphonuclear leukocytes modulate the production of tissue factor by monocytes via the release of free radicals. Activated platelets up-regulate tissue factor expression in vascular smooth muscle cells also via generation of free radicals. The complex of tissue factor plus coagulation factor VIIa activates the coagulation cascade converting fibrinogen to fibrin. Free radicals also inhibit fibrinolysis. Plasminogen activator inhibitor Type 1 (PAI-1) inhibits tissue plasminogen activator (tPA), which converts plasminogen into active plasmin. Tumor necrosis factor- $\alpha(\mathrm{TNF}-\alpha)$ stimulates PAI-1 production via generation of free radicals
DOI: 10.5530/ijopp.12.2.17

\section{Address for} correspondence: Anisha Thomas, Pharm.D Intern, Department of Pharmacy Practice, Bapuji Pharmacy College, Davangere, Karnataka, INDIA. Phone no: +91-7406116489 Email Id: anishathomas53@ gmail.com

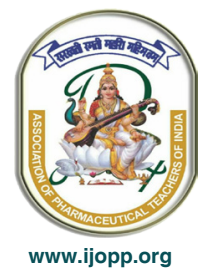


and free radical scavengers abolish the induction of PAI-1 by TNF- $\alpha$. Vascular endothelial cells also produce PAI-1 by interleukin-1 stimulation. Radical scavengers also abolish the increase of PAI-1 expression induced by interleukin-1. ${ }^{2}$ Therefore, the presence of free radical scavengers may improve the coagulation conditions in ischemic lesions.

Moreover, edaravone showed antioxidant properties as well as anti-inflammatory effects that reduced the induction of inducible nitric oxide synthase. Edaravone is thought to protect against ischemic brain edema based upon such experimental findings, but no anti-edema effect has been clearly identified in clinical studies.

\section{Current Treatment: Tissue Plasminogen Activator (tPA, Alteplase, Activase, Actilyse)}

The only FDA approved treatment for ALS is the thrombolytic, tissue plasminogen activator (tPA, Alteplase), a plasminogen activator that promotes thrombolysis by activating the endogenous fibrinolytic system. In brief, tPA catalyzes the conversion of plasminogen to plasmin, which in turn degrades fibrin and leads to clot lysis and cerebral reperfusion. Alteplase has been shown to be effective up to $4.5 \mathrm{~h}$ after a stroke, but is currently only Food and Drug Administration (FDA) approved for use within the first $3 \mathrm{~h}$ of a stroke in patients documenting the absence of an Intracerebral Haemorrhage (ICH). In alteplase-treated patients, the short-term incidence of symptomatic ICH is significantly higher in tPA-treated patients than in placebo-treated controls. ${ }^{3}$ Following the initial approval of alteplase treatment, the treatment was further developed and refined to a point where in the most recent ECASS III trial ${ }^{4}$ the investigators showed that tPA could be administered up to $4.5 \mathrm{~h}$ following a stroke without an increased incidence of mortality. However, the mortality rate did not differ significantly between the alteplase and placebo groups and there was no significant difference in the rate of other Serious Adverse Events (SAEs). Clearly, this is a major advancement in the stroke field; however, with only one effective FDA- approved stroke treatment there remains a need to develop treatments for stroke.

\section{Free Radicals as a Target}

Free radicals are a valid target for therapeutic intervention for the treatment of ALS, ${ }^{5}$ because oxidative stress is a major component of the ischemic stroke cascade, ${ }^{6,7}$ which is activated following vascular occlusion. However, the ischemic stroke cascade is a multi-component cascade with sequential activation of a complex series of pathophysiological events that evolve temporally. ${ }^{8}$

However, the therapeutic benefit of edaravone may be due to more than its antioxidant activity and may be directly related to its multi-target pharmacology and the ability of edaravone to regulate numerous signaling pathways. For example, recent mechanistic research has suggested that edaravone can suppress delayed neuronal death, counteract microglia-induced neurotoxicity and reduce the long-term inflammation. ${ }^{9}$ It has also been shown that edaravone can inhibit lipoxygenase, an enzyme responsible for lipid oxidation damage and could also directly suppress the oxidation of low-density lipoprotein. In addition to the attenuating the deleterious effects mediated by the ischemic cascade, it has been suggested that may prevent the development of edema following a stroke by inhibiting astrocyte production of expression of vascular endothelial growth factor. Moreover, Kituchi et al. hypothesized that edaravone may reduce aquaporin- 4 levels following an ischemic event, thereby reducing edema. Last, Yagi et al. showed that edaravone may reduce the activity of matrix metalloproteinase- 9 and further protect against vascular damage and Intracerebral Hemorrhage (ICH). Taken together, edaravone may prevent neuronal degeneration, vascular compartment damage and behavioral deficits resulting from both ischemia progression and edema. Thus, edaravone may fit into a class of multi-target compounds that may be useful to treat stroke.

\section{Mechanism of Edaravone}

Free radicals are important factors affecting both coagulation and thrombolysis systems in ischemic lesions. ${ }^{10}$ Free radicals affect these systems through various pathways (Figure 1). Free radicals activate coagulation.

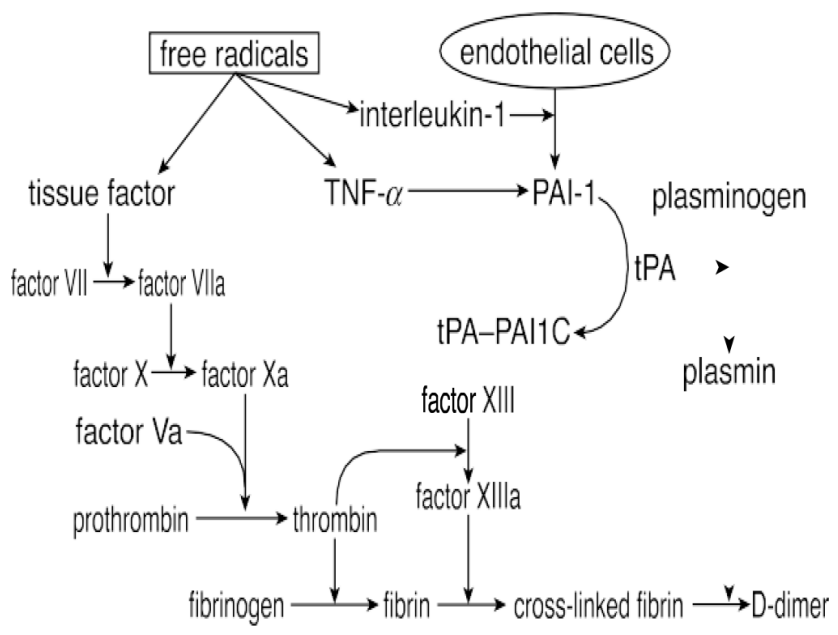

Figure 1: Mechanisms of free radicals affecting the pathways of coagulation and thrombolysis. PAI-1: plasminogen activator inhibitor type 1, TNF- $\alpha$ : tumor necrosis factor- $\alpha$, tPA: tissue plasminogen activator, tPA-PAl1C: tissue plasminogen activator-plasminogen activator inhibitor type 1 complex. 


\section{Future therapeutic perspectives}

The mechanisms of brain injury related to ischemia/ reperfusion and the neuroprotective mechanisms of action of edaravone, based on the in vitro and in vivo evidence described above, are schematically shown in Figure 2. Edaravone inhibits lipid peroxide accumulation by scavenging free radicals generated during brain ischemia/reperfusion and thereby attenuates vascular and neuronal cell injury. In addition, edaravone improves cerebral blood flow by increasing eNOS and, because of its antioxidant property, without increased production of peroxynitrite. It can be concluded that edaravone may attenuate vascular endothelial and neuronal injuries by inhibiting oxidative stress and by enhancing eNOS activity.

One major limitation of stroke therapy is the lag time from onset of stroke to the beginning of treatment because many cell death mechanisms start within minutes. Although peri-ischemic areas (penumbra) may be salvaged by recanalization, this treatment should be launched within 3-6 h after onset of stroke. Therefore, the number of patients eligible for thrombolytic therapy is, to begin with, limited. However, unlike with recanalization, there is a wide therapeutic time window for brain edema because the fluid accumulation in the brain progresses for days after onset of stroke. As reported recently, edaravone has excellent neuroprotective effects in the cortical area (but not in the subcortical area) regard less of cerebral blood flow. ${ }^{11}$ Cerebral cortex contains the major part of ischemic penumbra, in which cells are still only mildly damaged and may undergo apoptosis rather than necrosis. ${ }^{12}$ Therefore,

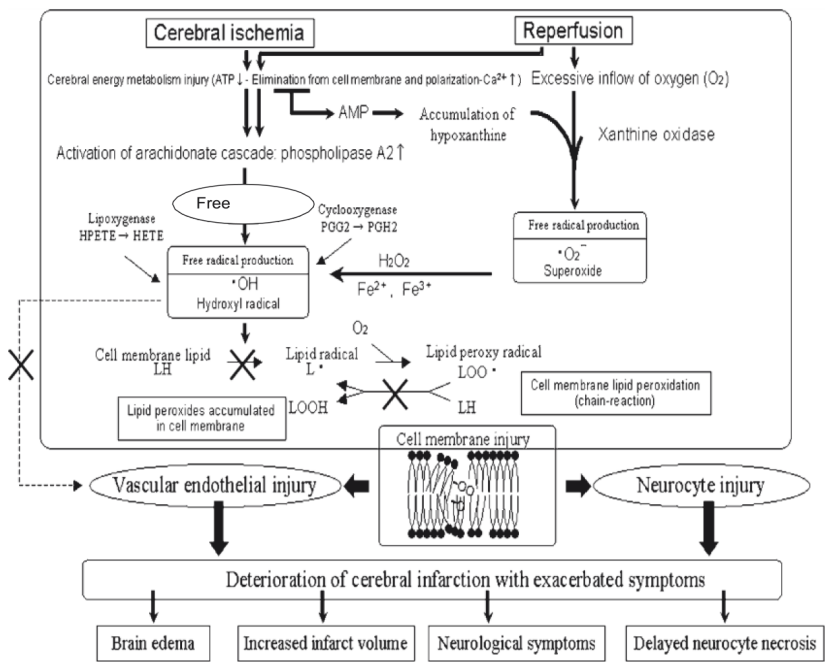

Figure 2: Development of brain injury during ischemia/reperfusion and proposed mechanisms of the neuroprotective action of edaravone. ATP: adenosine triphosphate; AMP: adenosine monophosphate; HPETE: hydroperoxyeicosatetraenoic acid; HETE, hydroxyeicosatetraenoic acid; PGG2: prostaglandin G2; PGH2: prostaglandin H2; L: alkyl; $x$ : site of the inhibitory effect of edaravone. cerebral cortex could be salvageable after ischemic events. ${ }^{13,14}$ In experimental models of focal brain ischemia infused with edaravone for $90 \mathrm{~min}$ its major oxidation product (2-oxo-3-(phenylbutazono)-butanoic acid, OPB) was found in the penumbra area. This finding indicated that edaravone is likely to protect brain by reacting with oxygen radicals formed in the penumbra. ${ }^{15}$

\section{CONCLUSION}

From the above review it is revealed that, use of edaravone appeared to be safe in acute ischemic stroke as there was no adverse reaction in any of patients though some studies reported insignificant skin rashes and abnormal liver function in the edaravone group. There are few case reports of acute renal failure and fulminant hepatitis associated with edaravone administration and therefore careful monitoring of renal and liver functions is warranted while using this drug. However, the results of the present study suggest that edaravone treatment may be safe and effective in providing early and sustained neurological improvement in patients with acute ischemic stroke. Larger randomized, double blind, case-controlled studies are required to confirm this. Free radicals play a crucial role in brain ischemic injury by exacerbating membrane damage through the peroxidation of unsaturated fatty acids of cell membrane, leading to neuronal death and brain edema. Edaravone is a potent free radical scavenger that has been used clinically to reduce neuronal damage following ischemic stroke. However, the most severe form of brain edema in ischemic stroke is observed when both, free radical mediated cell injury and protease-mediated matrix degradation, act in combination. Therefore, the beneficial effect of edaravone on ischemic stroke may be enhanced in combinations with other treatments. For example, a recent report has showed that combination therapy (edaravone plus tissue plasminogen activator) significantly increases the survival rate of rats with transient MCAO and reduces the infract volume and haemorrhage and that the neuroprotective effect of edaravone treatment is dependent on reduced accumulation of lipid peroxidation products. ${ }^{16}$ Post-reperfusion brain edema and hemorrhagic events induced by thrombolytic therapy may be reduced by free radical scavenging.

\section{ACKNOWLEDGEMENT}

We are grateful to Assistant Professor Dr. Akhila S Arjun, Department of Pharmacy Practice, Bapuji Pharmacy College for valuable help with the manuscript.

Indian Journal of Pharmacy Practice, Vol 12, Issue 2, Apr-Jun, 2019 


\section{CONFLICT OF INTEREST}

The authors declare no conflict of interest.

\section{ABBREVIATIONS}

ALS: Amyotrophic lateral sclerosis; ECASS III: European Cooperative Acute Stroke Study III; eNOS: Endothelial Nitric Oxide Synthase; MCAO: Middle Cerebral Artery Occlusion.

\section{REFERENCES}

1. Yoshida $H$, Yanai $H$, Namiki $Y$, Fukatsu-Sasaki $K$, Furutani $N$, Tada $N$ Neuroprotective effects of edaravone: A novel free radical scavenger. CNS Drug Rev. 2006;12(1):9-20.

2. Suda S, Igarashi H, Arai Y, Andou J, Chishiki T, Katayama Y. Effect of edaravone a free radical scavenger on ischemic cerebral edema assessed by Magnetic Resonance Imaging. Neurol Med Chir. 2007;47(5):197-201.

3. Tissue plasminogen activator for acute ischemic stroke. The National Institute of Neurological Disorders and Stroke rt-PA Stroke Study Group. N Engl J Med. 1995;333(24):1581-7. [PubMed: 7477192]

4. Hacke W, Kaste M, Bluhmki E, Brozman M, Davalos A, Guidetti D, et al. Thrombolysis with alteplase 3 to $4.5 \mathrm{~h}$ after acute ischemic stroke. N Engl J Med. 2008;359(13):1317-29. [PubMed: 18815396].

5. Lapchak PA. Translational stroke research using a rabbit embolic stroke model: a correlative analysis hypothesis for novel therapy development. Translational
Stroke Research. 2010;1(2):96-107. http://dx.doi.org/ 10.1007/s12975-0100018-4.

6. Lapchak PA, Araujo DM. Advances in ischemic stroke treatment: neuroprotective and combination therapies. Expert Opin Emerg Drugs. 2007;12(1):97-112. [PubMed: 17355216]

7. Love S. Oxidative stress in brain ischemia. Brain Pathol. 1999;9(1):119-31. [PubMed: 9989455]

8. Dirnagl U, ladecola C, Moskowitz MA. Pathobiology of ischaemic stroke: An integrated view. Trends Neurosci. 1999;22(9):391-7. [PubMed: 10441299]

9. Lee BJ, Egi Y, Leyen KV, Lo EH, Arai K. Edaravone, a free radical scavenger, protects components of the neurovascular unit against oxidative stress in vitro. Brain Res. 2010;1307:22-7. [PubMed: 19840779]

10. Flamm ES, Demopoulos HB, Seligman ML, Poser RG, Ransohoff J. Free radicals in cerebral ischemia. Stroke. 1978;9(5):445-7.

11. Amemiya S, Kamiya T, Nito C, et al. Anti-apoptotic and neuroprotective effects of edaravone following transient focal ischemia in rats. Eur J Pharmacol. 2005;516(2):125-30.

12. Li Y, Chopp M, Jiang N, Zhang ZG, Zaloga C. Induction of DNA fragmentation after 10 to $120 \mathrm{~min}$ of focal cerebral ischemia in rats. Stroke. 1995;26(7):1252-8.

13. Hossmann KA. Viability threshold and the penumbra of focal ischemia. Ann Neurol. 1994;54:271-84

14. Morimoto T, Globus MY, Busto R, Martinez E, Ginsberg MD. Simultaneous measurement of salicylate hydroxylation and glutamate release in the penumbra cortex following transient middle cerebral artery occlusion in rats. J Cereb Blood Flow Metab. 1996;16(1):92-9.

15. Kawai H, Nakai H, Suga M, Yuki S, Watanabe T, Saito K. Effects of a novel free radical scavenger, $\mathrm{MCl}-186$, on ischemia brain damage in rat distal middle cerebral artery occlusion model. J Pharmacol Exp Ther. 1997;281(2):921-7.

16. Zhang W, Sato K, Hayashi T, et al. Extension of ischemic therapeutic time window by a free radical scavenger, edaravone, reperfused with IPA in rat brain. Neurol Res 2004;26(3):342-8. 